Materials and Methods Patients were divided into three groups: current smoker, previous smoker (defined as having quit greater than 6 months prior to the ischemic event), and non-smoker. NIHSS scores were recorded upon admission and at discharge. The change in NIHSS score was defined as score at admission minus the score at discharge. Infarct and cerebral edema volumes were measured. Linear regression analysis was performed based on infarct or edema volume versus the change in NIHSS score.

Results Eighty-eight adult subjects ( $>18$ years) were included in the study, of which 48 (55\%) were female. Median age was 69 years (25-101). Twenty-three (26\%) were active smokers, 14 (16\%) were previous smokers, and 51 (58\%) were non-smokers. Mean infarct time, or mean time from last known well, was $508 \pm 347$ minutes. Mean infarct volume was $67,882 \pm 90,632 \mathrm{~mm}^{3}$. Mean edema volume was $69,432 \pm 93,878 \mathrm{~mm}^{3}$. Mean NIHSS score on admission was $16.9 \pm 7.5$ and mean NIHSS score at discharge was $8.9 \pm$ 8.4. Mean age at time of infarct for the smoking population was 10 years earlier when compared to the nonsmoking population, 61.9 versus 71.6 years. Linear regression was significant $(\mathrm{p}<0.0001)$ when comparing infarct or edema volume versus change in NIHSS score for current smokers only.

Conclusion On average, smokers had stroke 10 years earlier than non-smokers, which is consistent with previously published findings. Smokers also had a statistically significant correlation between infarct or edema volume and worsening change in NIHSS score, when compared to both previous smokers and non-smokers who had no significant correlation. These findings are valuable in patient counseling for smoking cessation. Patients who continue to smoke are more likely to have a stroke earlier in life with potentially more significant disability and worse prognosis.

Disclosures K. Swafford: None. J. Frank: None. J. Roberts: None. A. Trout: None. S. Grupke: None. K. Pennypacker: 6; C; co-owner of Cerelux, LLC. J. Fraser: 2; C; Stream Biomedical, Medtronic, Penumbra. 4; C; Cerelux, LLC, Fawkes Biotechnology. 6; C; owner of Cerelux, LLC, owner of Fawkes Biotechnology.

\section{E-111 INTRACRANIAL CAROTID RUPTURE DURING THROMBECTOMY WITH EFFECTIVE TANDEM OCCLUSION}

${ }^{1} \mathrm{E}$ Cora*, ${ }^{2} \mathrm{~A}$ Arthur, ${ }^{1} \mathrm{M}$ Schmidt, ${ }^{1} \mathrm{R}$ Vandorpe, ${ }^{3} \mathrm{~B}$ Drake, ${ }^{4} \mathrm{D}$ lancu, ${ }^{3} \mathrm{H}$ Lesiuk, ${ }^{1} \mathrm{D}$ Volders, ${ }^{1} \mathrm{~T}$ Huynh. ${ }^{1}$ Department of Diagnostic Radiology, QEIl Health Sciences Centre, Halifax, NS, Canada; ${ }^{2}$ Semmes-Murphey Clinic, Memphis, TN; ${ }^{3}$ Department of Neurosurgery, The Ottawa Hospital, Ottawa, ON, Canada; ${ }^{4}$ Department of Diagnostic Radiology, The Ottawa Hospital, Ottawa, ON, Canada

\subsection{6/neurintsurg-2020-SNIS.144}

Introduction Mechanical thrombectomy has become the standard of care for patients presenting with acute ischemic stroke due to a large vessel occlusion. Intracranial hemorrhage is a serious complication which may occur during this procedure. The more commonly recognized cause of intraprocedural intracranial hemorrhage is due to iatrogenic vessel perforation. Here we present a series of patients with terminal ICA occlusions and proximal carotid stenosis that had mechanical thrombectomy complicated by devastating subarachnoid hemorrhage. Similarities between cases are highlighted and we hypothesize on the possible mechanism.

Case Description Three patients presented acutely with stroke symptoms due to an intracranial large vessel occlusion and had thrombectomy performed. Two of these patients had a tandem proximal and distal ICA occlusion extending to the M1 MCA with stenosis at the origin of the ICA. During the mechanical thrombectomy procedure all three patients had significant subarachnoid hemorrhage originating from the distal intracranial ICA in the region of the distal dural ring. The patient who did not have stenosis at the origin of the ICA, had the procedure performed with a balloon guiding catheter high in the cervical ICA which was relatively occlusive. Two patients had coiling performed to stop the hemorrhage, while the third patient was deemed for palliation. All three patients passed away within days after this serious complication.

Results We hypothesize that the cause of the subarachnoid hemorrhages in these patients was due to performing angiography in a closed system with the proximal stenosis/balloon guiding catheter causing blockage of the proximal ICA and the ICA terminus thrombus blocking the distal aspect. When performing an angiographic run, we hypothesize that the

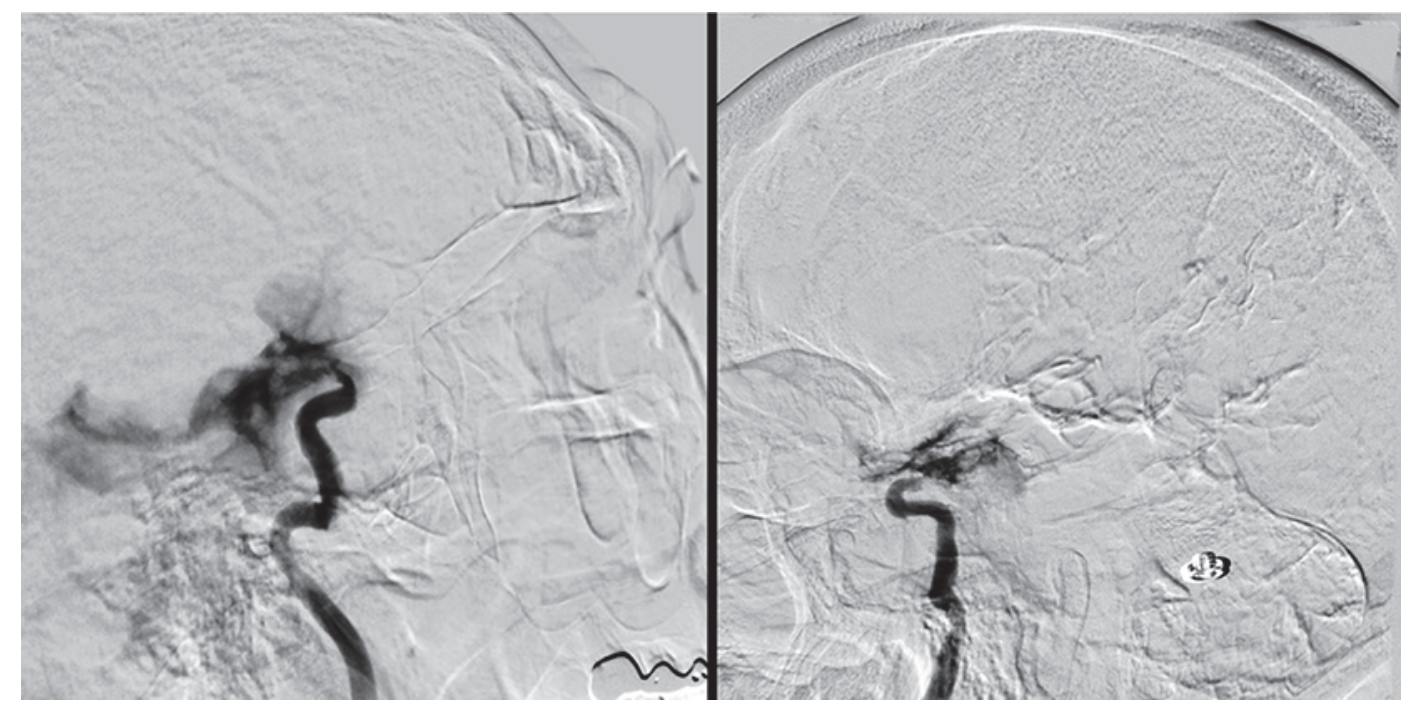

Abstract E-111 Figure 1 
increased pressure can lead to rupture of the arterial wall at the weakest spot, which may be just past the distal dural ring where the ICA travels intracranially within the subarachnoid space.

Conclusion We highlight a newly encountered mechanism for potential injury to the distal ICA during mechanical thrombectomy. Sudden increase in pressure affecting the ICA in a system that is closed when performing angiography can rupture the distal ICA as it goes intracranially resulting in subarachnoid hemorrhage. It is important to be aware of this fatal complication when performing mechanical thrombectomy in patients with ICA terminus occlusion especially with proximal tandem carotid stenosis. Awareness of this complication will help operators to avoid performing angiographic runs in this potential closed space if not deemed necessary to avoid this potentially devastating complication.

Disclosures E. Cora: None. A. Arthur: None. M. Schmidt: None. R. Vandorpe: None. B. Drake: None. D. Iancu: None. H. Lesiuk: None. D. Volders: None. T. Huynh: None.

\section{E-113 MIDDLE MENINGEAL ARTERY EMBOLIZATION FOR TREATMENT OF CHRONIC SUBDURAL HEMATOMA: A PROSPECTIVE INSTITUTIONAL CASE SERIES}

${ }^{1} \mathrm{~L}$ Rinaldo*, ${ }^{2} \mathrm{H}$ Cloft, ${ }^{3} \mathrm{~W}$ Brinjikji. ' Neurosurgery, Mayo Clinic, Rochester, MN; ${ }^{2}$ Radiology, Mayo Clinic, Rochester, MN; ${ }^{3}$ Radiology and Neurosurgery, Mayo Clinic, Rochester, MN

\subsection{6/neurintsurg-2020-SNIS.145}

Background Embolization of the middle meningeal artery (MMA) has emerged a potential treatment for chronic subdural hematoma (cSDH), either as a stand-alone therapy or an adjunct to surgical evacuation.

Methods Information on patients with a cSDH at least 10 $\mathrm{mm}$ in maximal thickness selected for MMA embolization was entered into a prospectively collected database. The embolization procedure consisted of MMA catheterization followed by injection of 150-250 $\mu \mathrm{m}$ polyvinyl alcohol (PVA) particles until occlusion of the MMA distal to the sphenoid ridge. For patients who also underwent surgical evacuation, MMA embolization was performed after surgery in all cases. Descriptive statistics of treatment outcomes, which included $\mathrm{cSDH}$ size on final head CT and the incidence of cSDH surgical evacuation after MMA embolization, are provided.

Results MMA embolization was performed in 23 patients with 29 cSDHs. The mean age of the cohort was 72.3 years [Standard deviation $(\mathrm{SD})=9.2$ ] and a majority of patients were male $(n=24,82.8 \%)$. History of anti-coagulation use was present in 14 patients (60.9\%). Mean maximal dimension and associated midline shift of treated cSDHs were 18.1 $(\mathrm{SD}=4.9)$ and $4.6 \mathrm{~mm}(\mathrm{SD}=3.9)$. Surgical evacuation prior to MMA embolization was performed for 12 cases (41.4\%). A thromboembolic complication during MMA embolization resulting in a new minor neurologic deficit occurred in a single patient (4.3\%). Overall, 27 cSDHs (93.1\%) were reduced in size on final follow-up CT, and 10 were completely resolved (34.4\%). Stratified by initial medical versus surgical management, $88.2 \% \quad(n=15 / 17)$ and $100.0 \% \quad(n=12 / 12)$ of cSDHs were reduced in size and $33.3 \%(n=4 / 12)$ and $35.3 \%$ $(n=6 / 17)$ were completely resolved on last follow-up CT. Two cSDHs $(6.9 \%)$ in a single patient initially treated with surgery required repeat surgical evacuation after MMA embolization.
Mean time between MMA embolization and last follow-up head CT was 3.7 months $(\mathrm{SD}=3.1)$.

Conclusion Our preliminary results suggest MMA embolization may be an effective treatment for cSDHs. Randomized trials are indicated.

Disclosures L. Rinaldo: None. H. Cloft: None. W. Brinjikji: None.

\section{E-114 THE USE OF CONE-BEAM CT NEEDLE GUIDANCE FOR PERCUTANEOUS SCLEROTHERAPY OF AIRWAY VENOUS MALFORMATIONS}

W Brinjikji*. Radiology, Mayo Clinic, Rochester, MN

\subsection{6/neurintsurg-2020-SNIS.146}

Background and Purpose Venous malformations involving the posterior pharyngeal wall, hypopharynx and periglottic regions can result in substantial morbidity due to chronic and acute airway obstruction. Percutaneous sclerotherapy for management of these patients is challenging due to the proximity of these malformations to vital structures including the carotid and vertebral arteries. We retrospectively reviewed our institutional case series of patients who received sclerotherapy for airway venous malformations using a novel cone-beam CT needle guidance software.

Methods Following institutional review board approval, all patients with craniofacial venous malformations involving airway structures with the assistance of Philips Xper-Guide Cone Beam CT needle guidance were identified. We performed all interventions under general endotracheal anesthesia and the malformations were involving the airway. In general, patients were given an $8 \mathrm{mg}$ dose of dexamethasone immediately prior to sclerotherapy. An XperCT was performed and XperGuide needle guidance was activated. Targets were chosen and using the needle guidance a 20 or $22 \mathrm{G}$ spinal needle was advanced into the malformation. After confirmation of needle positioning with a venogram, Bleomycin (1 mg/1 mL) was injected slowly into the malformation using a negative roadmap technique. Following treatment, patients had airway monitoring in the ICU for a minimum of 4 hours following sclerotherapy and if no substantial swelling or airway compromise was noted, patients were extubated. Patients were generally sent home with a 5 day course of steroids to prevent swelling of the malformation following sclerotherapy. All patients received follow-up at 1 week, 6 weeks and 3 months. Studied outcomes included technical success rate, procedural complications and symptom improvement.

Results Over the past twelve months, a total of 12 consecutive patients received 20 sessions of bleomycin sclerotherapy for treatment of airway venous malformations with the assistance of Cone-Beam CT Needle Guidance (XperGuide, Philips). The most common indications for treatment were obstructive sleep apnea refractory to CPAP (6 patients) and difficulty swallowing solid foods (6 patients) For these 20 sessions, a total of 54 different venous malformations were targeted. In all cases, either $20 \mathrm{G}$ or $22 \mathrm{G}$ spinal needles were successfully advanced into the venous malformations and bleomycin sclerotherapy was administered. Thus, the technical success rate was $100 \%$. There were no hemorrhagic or ischemic complications related to the sclerotherapy. In 19/20 sessions, patients were successfully extubated the day of the procedure. One patient required an additional day of intubation due to swelling of a 